\title{
Volumetric modulated arc radiotherapy of the whole larynx, followed by a single affected vocal cord, for T1a glottic cancer: Dosimetric analysis of a case
}

\author{
SEUNG-GU YEO \\ Department of Radiation Oncology, Soonchunhyang University College of Medicine, \\ Soonchunhyang University Hospital, Cheonan, Chungnam 330-721, Republic of Korea
}

Received July 21, 2015; Accepted December 3, 2015

DOI: $10.3892 / \mathrm{mco} .2016 .732$

\begin{abstract}
Radiation therapy (RT) and endolaryngeal surgery are standard treatments for early-stage glottic cancer. They have closely matched oncological outcomes; however, it is debatable which method is superior in terms of functional outcomes. Several dosimetric studies have demonstrated that, compared with conventional RT, intensity-modulated RT (IMRT) reduces unnecessary radiation of the adjacent normal tissues, including the carotid artery and thyroid gland. However, RT targets the whole larynx, whereas endolaryngeal surgery is a highly focused treatment involving the en bloc resection of a tumor with safety margins. For T1a glottic cancer, in which the tumor is limited to one vocal cord, the technical feasibility of targeting IMRT on the single vocal cord affected has been investigated; however, the clinical feasibility and the possibility of inferior local control remain to be elucidated. In the present case study, IMRT was used to treat the whole larynx first, and then to treat a single vocal cord. The patient in the present study had T1a glottic cancer, and received volumetric modulated arc therapy with a total dose of $63 \mathrm{~Gy} / 28$ fractions. The first treatment phase ( $40.5 \mathrm{~Gy} / 18$ fractions) targeted the whole larynx to eliminate subclinical disease. The second treatment phase (22.5 Gy/10 fractions) targeted only the involved vocal cord. During this treatment phase, the exposure of the non-involved right vocal cord, the right carotid artery and the thyroid gland to the radiation was lower compared with the continuation of the initial treatment approach. These findings suggested that changing the target volume from the whole larynx to the affected vocal cord during the course
\end{abstract}

Correspondence to: Dr Seung-Gu Yeo, Department of Radiation Oncology, Soonchunhyang University College of Medicine, Soonchunhyang University Hospital, 31 Soonchunhyang 6-gil, Cheonan, Chungnam 330-721, Republic of Korea

E-mail: md6630@schmc.ac.kr

Key words: glottic cancer, larynx, volumetric modulated arc therapy, intensity modulated radiotherapy, target volume of IMRT is feasible for T1a glottic cancer, and that it may reduce functional side effects while maintaining oncological outcomes.

\section{Introduction}

Due to the involvement of the vocal folds, patients with glottic cancer often present with hoarseness in the early stages of the disease (T1-2N0M0). Approximately $75 \%$ of patients with glottic cancer are diagnosed early (1). For early-stage glottic cancer, two treatment options exist: radiation therapy (RT) and endolaryngeal surgery (with or without a laser). The two modalities offer excellent and comparable oncological outcomes (2); therefore, functional outcomes and toxicity profiles are the predominant considerations used to develop a therapeutic regimen (3).

Endolaryngeal surgery involves the en bloc resection of a tumor with a safety margin (4). In contrast, the target volume in RT encompasses the entire larynx, even for Tla glottic cancer, in which the entire tumor is limited to one vocal cord (5). To decrease the irradiation of adjacent non-cancerous tissues, the use of intensity-modulated RT (IMRT) for early-stage glottic cancer patients has been recommended (6-8). However, to date, few studies have investigated the possibility of changing the target volume. Only one institution in the Netherlands has investigated limiting the IMRT target volume to a single affected vocal cord for T1a glottic cancer (9-14); their studies revealed that IMRT with a limited target volume was associated with a reduction in the irradiation of adjacent normal tissue. However, the oncological outcomes of limiting the target volume to a single vocal cord throughout the course of RT remain to be elucidated.

In a similar manner to routine fractionated RT for other tumor entities (15), it was hypothesized that limiting the target volume to a single vocal cord for patients with T1a glottic cancer may be appropriate if it is performed following the completion of irradiation for subclinical microscopic disease in the larynx. In the present study, the results of a dosimetric analysis of a patient with T1a glottic cancer are shown, whose IMRT target volume was reduced from the whole larynx to the single vocal cord affected in the second phase of treatment. 


\section{Case report}

A 59-year-old male was admitted to hospital complaining of hoarseness. A laryngoscopic examination revealed a luminal protruding mass on the left vocal cord. Neck computed tomography $(\mathrm{CT})$ revealed a contrast-enhancing mass on the left vocal cord without cervical lymph node enlargement. The tumor was limited to the left vocal cord, and the vocal cord mobility remained intact. The pathological analysis of a laryngoscopy biopsy specimen revealed a moderately differentiated squamous cell carcinoma. No evidence of distant metastasis was identified using ${ }^{18} \mathrm{~F}$-fluorodeoxyglucose positron emission tomography-CT. The clinical tumor-lymph node-metastasis stage was defined as T1aNOM0 glottic cancer, according to the American Joint Committee on Cancer Staging Manual Seventh Edition (16). The present study was performed in accordance with the guidelines of our institutional review board; written informed consent was waived, since this is a case report with retrospective dosimetric analysis.

For RT simulation, the patient was immobilized in the supine position with a thermoplastic head and neck mask. A contrast-enhanced planning CT scan was performed using a 16-slice CT scanner (Brilliance CT Big Bore; Philips Medical Systems, Cleveland, $\mathrm{OH}, \mathrm{USA}$ ) with a $0.2-\mathrm{cm}$ slice thickness. The initial clinical target volume (CTV) included the false and true vocal cords, anterior and posterior commissures, arytenoids and subglottic region, extending from the superior thyroid notch to the bottom of the cricoid cartilage. The planning target volume (PTV) was created by adding a $0.5-\mathrm{cm}$ isotropic margin around the CTV. The contoured organs at risk (OAR) included the carotid artery, whole thyroid gland and spinal cord. The right and left common, internal and external carotid arteries were delineated from the sternoclavicular joints, and upward to the base of the skull.

A volumetric modulated arc therapy (VMAT) plan was created using the Eclipse Treatment Planning system (Varian Medical Systems, Inc., Palo Alto, CA, USA), as previously described (17). A double-arc plan was generated using 6-MV photons and a high-definition multileaf collimator. The plan was normalized in order that $\geq 95 \%$ of the PTV received $100 \%$ of the prescribed dose. The prescribed dose in the initial plan was 40.5 Gy at 2.25 Gy per fraction. Prior to the end of the initial treatment session, a second planning CT scan was obtained, and only the affected left vocal cord was contoured as the CTV. The PTV was defined as the CTV plus a $0.5-\mathrm{cm}$ margin. The prescribed dose of the reduced field (RF) volumetric modulated double-arc plan was 22.5 Gy in 10 fractions. Overall, the total prescribed dose was $63 \mathrm{~Gy}$ in 28 fractions. The treatment was performed using the Novalis Tx platform (Varian Medical Systems, Inc./BrainLab, Feldkirchen, Germany). The set-up was verified daily prior to treatment with ExacTrac X-ray monitoring (BrainLab), and weekly by cone-beam CT. The patient completed the scheduled treatment without interruption. During the course of treatment, the patient developed acute mild dysphagia, which subsided spontaneously.

Figs. 1 and 2 show the isodose distribution and the dose-volume histogram of the OAR, respectively, according to the target volume (initial or RF plan). To analyze the dosimetric advantages of the RF plan, the prescribed dose in the initial plan was modified to be identical with that of the RF plan (22.5 Gy/10 fractions). Table I shows that the adoption of the RF plan, compared with the initial plan, lowered the mean and maximum doses to the OAR.

\section{Discussion}

Unlike other head and neck cancers (18), the application of IMRT (including VMAT) is not generalized in early-stage glottic cancer. Conventional RT delivered with lateral opposed beams is more commonly used for early-stage glottic cancer due to the small RT field and the lack of requirement for elective cervical node irradiation (17). However, several institutions have demonstrated that IMRT is able to reduce unnecessary irradiation of the carotid artery, which may cause carotid artery stenosis and ischemic stroke (6-8). Furthermore, it was demonstrated that IMRT is able to reduce irradiation not only to the carotid artery, but also to the thyroid gland, which is able to induce hypothyroidism (17). It was proposed that an increased conformality with IMRT and organ motion may increase the risk of marginal failures (19). However, a previous clinical study of IMRT for early-stage glottic cancer reported that the local control outcomes were comparable with those with conventional RT $(20,21)$.

Another aspect of RT that should be investigated is the target volume range used for early-stage glottic cancer. Endolaryngeal surgery is a highly focused treatment for a gross tumor with safety margins (4), whereas the RT field encompasses the whole larynx (5). Targeting only the involved vocal cord with RT could further improve voice quality, and reduce the radiation exposure of the surrounding OAR. Osman et al (9-12), Levendag et al (13) and Kwa et al (14) explored the technical feasibility, and dosimetric advantages, of targeting only a single affected vocal cord with IMRT for Tla glottic cancer.

However, the clinical outcomes resulting from the adoption of this major change in the target volume remain to be elucidated. Janssen et al (22) reported a three-year local control rate of $95 \%$ for 41 patients with T1-2 glottic cancer, among whom 31 had received IMRT in which the target was restricted to the involved laryngeal side. The extent of local control appears to be high, although the present study did not report the local recurrence rate according to the target volume range, or the site of local recurrence. In addition, concurrent systemic chemotherapy has been administered to several patients with T2 tumors. Zumsteg et al (20) reported conventional RT $(n=282)$ and IMRT $(n=48)$ outcomes for patients with T1-2N0M0 glottic cancer. The IMRT target volume was the entire larynx. In total, 14 of 41 patients had recurrent bilateral vocal cord disease, although the tumor presentation (single or bilateral) was not specified. Lim et al (5) analyzed patterns of local failure following conventional RT for 222 patients with T1-2N0M0 glottic cancer. The local relapse sites of 29 patients who presented with a tumor confined to a single vocal cord included 28 cases of a tumor of the ipsilateral vocal cord, and one case of a tumor in the subglottis. The authors speculated that the absence of recurrence involving the contralateral vocal cord was due to the fact that the target volume involved the whole larynx.

Until the clinical feasibility of single vocal cord RT is verified, reducing the RT target volume to a single vocal cord 
Table I. Dosimetric comparison by target volume (planning target volume dose prescription=22.5 Gy/10 fractions).

\begin{tabular}{llrc}
\hline & & \multicolumn{2}{c}{ Target volume } \\
\cline { 3 - 4 } Tissue/gland & Dose (Gy) & Whole larynx & The involved left vocal cord \\
& & 23.6 & 20.7 \\
Vocal cord, right & Mean & 24.3 & 24.0 \\
& Maximum & 3.4 & 1.6 \\
Carotid artery, right & Mean & 16.3 & 7.6 \\
& Maximum & 2.9 & 1.0 \\
Thyroid gland & Mean & 25.3 & 18.5 \\
& Maximum & & \\
\hline
\end{tabular}

A

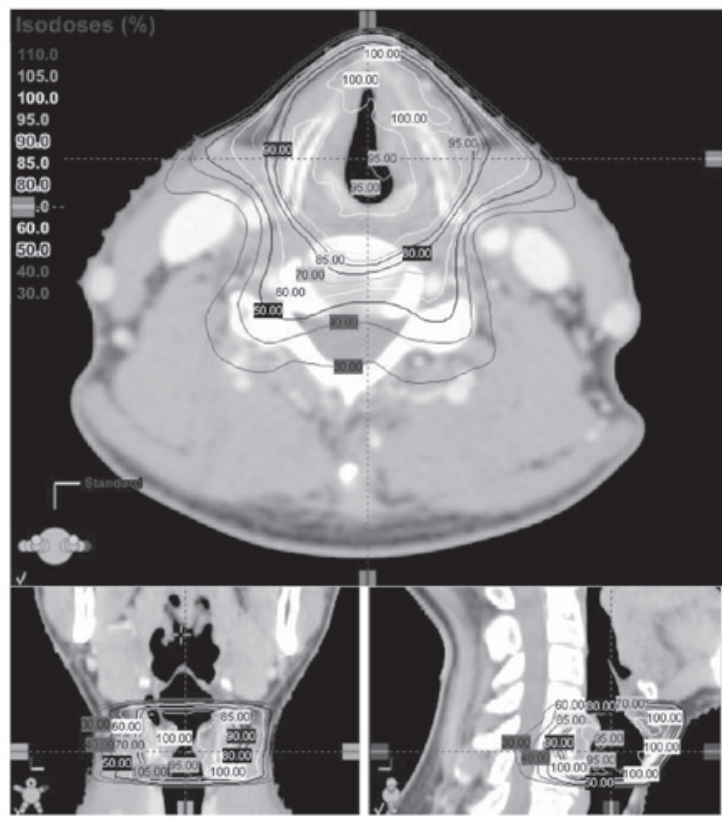

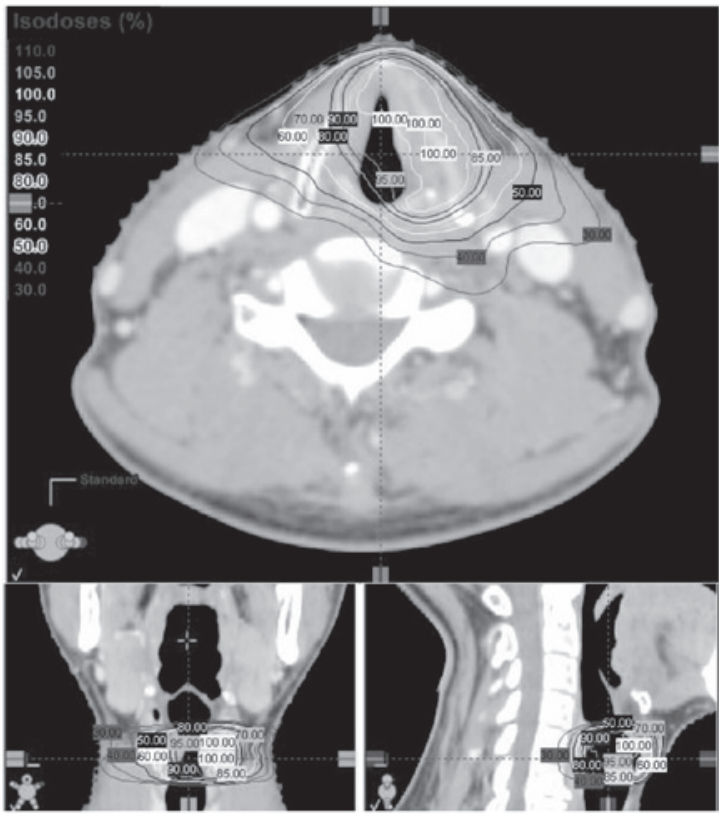

Figure 1. Comparison of the dose distribution (isodose lines $\geq 30 \%$ ) by target volume: (A) whole larynx, and (B) the involved vocal cord. Transverse (top), coronal (bottom left) and sagittal (bottom right) images are shown.

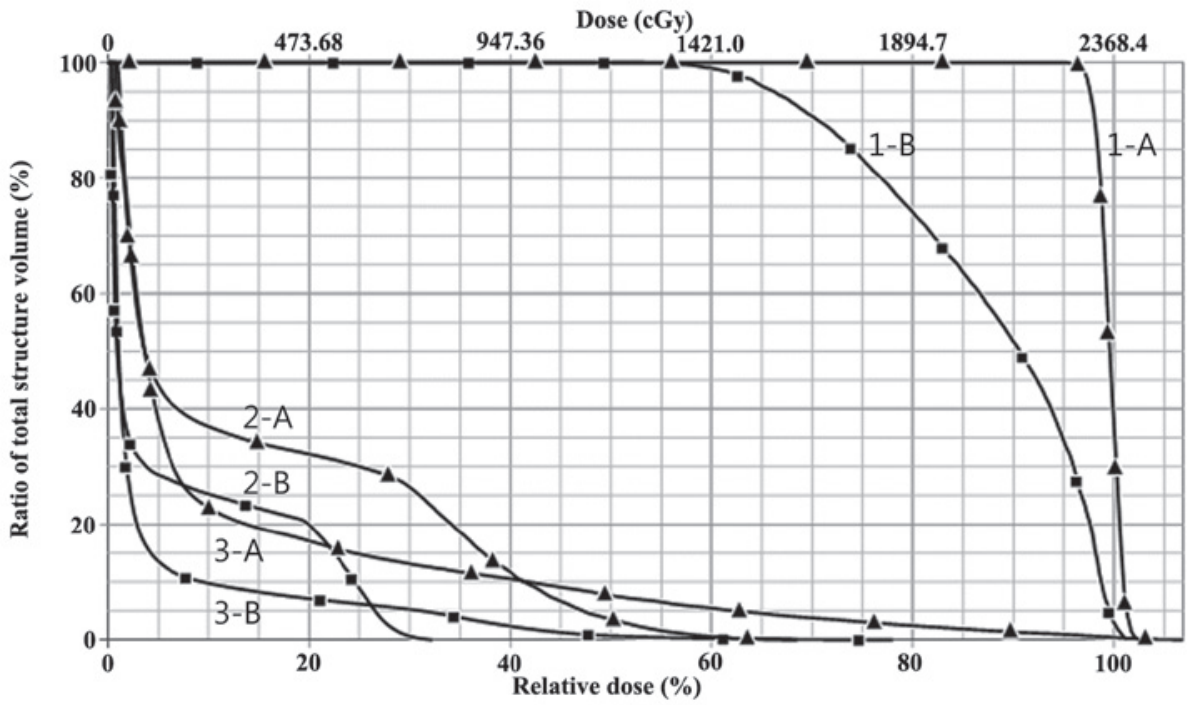

Figure 2. Dose-volume histogram according to the target volume: (A) of the whole larynx; and (B) of the involved vocal cord. The numbers in the histogram denote: (1) the non-involved right vocal cord; (2) the right carotid artery; and (3) the thyroid gland. The planning target volume dose prescription was $22.5 \mathrm{~Gy} / 10$ fractions. 
may be permissible if performed subsequently to completion of the irradiation necessary to sterilize subclinical disease in the larynx. Generally, a dose of 45-50 Gy in 2 Gy fractions is necessary to sterilize subclinical microscopic tumor cells (15). In the present study, the patient began RF treatment following initial treatments of $40.5 \mathrm{~Gy} / 18$ fractions, considering a fraction dose of 2.25 Gy and incidental irradiation of the contralateral vocal cord during single vocal cord RT. During RF treatment, the radiation dose administered to the surrounding normal tissues, including the contralateral vocal cord, carotid artery and thyroid gland, was reduced compared with continuation of the initial IMRT plan (Figs. 1 and 2, and Table I). The normal tissue complication probability model of radiation-induced hypothyroidism indicated that the probability of hypothyroidism increases with a higher mean dose to the thyroid gland (odds ratio, 1.064/Gy) (23). In a previous study, VMAT exhibited a median 8 Gy reduction in mean thyroid dose compared with conventional RT (17). The RF plan used in the present study decreased the mean thyroid dose by an additional $2 \mathrm{~Gy}$, and thus may have helped to minimize the risk of hypothyroidism. Furthermore, dose reduction to the vocal cord and the carotid artery is anticipated to improve voice quality and decrease the risk of cerebrovascular events, respectively.

A second planning CT scan and re-planning for RF treatment is also beneficial with respect to the concept of adaptive IMRT (24). The continuous use of an initial, highly conformal IMRT plan with no adjustment may increase the risk of marginal failure and toxicity, when the morphology of the target and the OAR change markedly during a treatment course with a duration of several weeks.

In conclusion, the present case study suggested that RF re-planning with a change in target volume from the whole larynx to the affected vocal cord during the latter course of IMRT for T1a glottic cancer may successfully be adopted. By following this scheme, functional outcomes ought to be improved, while exerting no detrimental effects on the already excellent oncological outcome.

\section{Acknowledgements}

This study was supported by the Soonchunhyang University Research Fund.

\section{References}

1. Warner L, Chudasama J, Kelly CG, Loughran S, McKenzie K Wight R and Dey P: Radiotherapy versus open surgery versus endolaryngeal surgery (with or without laser) for early laryngeal squamous cell cancer. Cochrane Database Systematic Rev 12: CD002027, 2014

2. Yoo J, Lacchetti C, Hammond JA and Gilbert RW; Head and Neck Cancer Disease Site Group: Role of endolaryngeal surgery (with or without laser) versus radiotherapy in the management of early (T1) glottic cancer: A systematic review. Head Neck 36: 1807-1819, 2014.

3. van Loon Y, Sjügren EV, Langeveld TP, Baatenburg de Jong RJ, Schoones JW and van Rossum MA: Functional outcomes after radiotherapy or laser surgery in early glottic carcinoma: A systematic review. Head Neck 34: 1179-1189, 2012.

4. Remacle M, Eckel HE, Antonelli A, Brasnu D, Chevalier D, Friedrich G, Olofsson J, Rudert HH, Thumfart W, de Vincentiis M and Wustrow TP: Endoscopic cordectomy. A proposal for a classification by the working committee, European laryngological society. Eur Arch Otorhinolaryngol 257: 227-231, 2000
5. Lim YJ, Wu HG, Kwon TK, Hah JH, Sung MW, Kim KH and Park CI: Long-term outcome of definitive radiotherapy for early glottic cancer: Prognostic factors and patterns of local failure. Cancer Res Treat 47: 862-870, 2015

6. Gomez D, Cahlon O, Mechalakos J and Lee N: An investigation of intensity-modulated radiation therapy versus conventional two-dimensional and 3D-conformal radiation therapy for early stage larynx cancer. Radiat Oncol 5: 74, 2010.

7. Rosenthal DI, Fuller CD, Barker JL Jr, Mason B, Garcia JA, Lewin JS, Holsinger FC, Stasney CR, Frank SJ, Schwartz DL, et al: Simple carotid-sparing intensity-modulated radiotherapy technique and preliminary experience for T1-2 glottic cancer. Int J Radiat Oncol Biol Phys 77: 455-461, 2010.

8. Chera BS, Amdur RJ, Morris CG and Mendenhall WM: Carotid-sparing intensity-modulated radiotherapy for early-stage squamous cell carcinoma of the true vocal cord. Int J Radiat Oncol Biol Phys 77: 1380-1385, 2010.

9. Osman SO, de Boer HC, Heijmen BJ and Levendag PC: Four-dimensional CT analysis of vocal cords mobility for highly focused single vocal cord irradiation. Radiother Oncol 89: 19-27, 2008.

10. Osman SO, de Boer HC, Astreinidou E, Gangsaas A, Heijmen BJ and Levendag PC: On-line cone beam CT image guidance for vocal cord tumor targeting. Radiother Oncol 93: 8-13, 2009.

11. Osman SO, Astreinidou E, de Boer HC, Keskin-Cambay F, Breedveld S, Voet P, Al-Mamgani A, Heijmen BJ and Levendag PC: IMRT for image-guided single vocal cord irradiation. Int J Radiat Oncol Biol Phys 82: 989-997, 2012.

12. Osman SO, Astreinidou E, Levendag PC and Heijmen BJ: Impact of geometric variations on delivered dose in highly focused single vocal cord IMRT. Acta Oncol 53: 278-285, 2014.

13. Levendag PC, Teguh DN, Keskin-Cambay F, Al-Mamgani A, van Rooij P, Astreinidou E, Kwa SL, Heijmen B, Monserez DA and Osman SO: Single vocal cord irradiation: A competitive treatment strategy in early glottic cancer. Radiother Oncol 101: 415-419, 2011.

14. Kwa SL, Al-Mamgani A, Osman SO, Gangsaas A, Levendag PC and Heijmen BJ: Inter- and intrafraction target motion in highly focused single vocal cord irradiation of T1a larynx cancer patients. Int J Radiat Oncol Biol Phys 93: 190-195, 2015.

15. Withers HR, Peters LJ and Taylor JM: Dose-response relationship for radiation therapy of subclinical disease. Int J Radiat Oncol Biol Phys 31: 353-359, 1995.

16. Edge SB, Byrd DR, Compton CC, Fritz AG, Greene FL and Trotti A (eds): AJCC cancer staging manual. 7th edition. Springer, New York, NY, 2010.

17. Kim ES and Yeo SG: Volumetric modulated arc radiotherapy sparing the thyroid gland for early-stage glottic cancer: A dosimetrical analysis. Oncology Lett 7: 1987-1991, 2014.

18. Kong M, Hong SE, Choi J and Kim Y: Comparison of survival rates between patients treated with conventional radiotherapy and helical tomotherapy for head and neck cancer. Radiat Oncol J 31: $1-11,2013$.

19. Feigenberg SJ, Lango $M$, Nicolaou $N$ and Ridge JA: Intensity-modulated radiotherapy for early larynx cancer: Is there a role? Int J Radiat Oncol Biol Phys 68: 2-3, 2007.

20. Zumsteg ZS, Riaz N, Jaffery S, Hu M, Gelblum D, Zhou Y, Mychalczak B, Zelefsky MJ, Wolden S, Rao S and Lee NY: Carotid sparing intensity-modulated radiation therapy achieves comparable locoregional control to conventional radiotherapy in T1-2N0 laryngeal carcinoma. Oral Oncol 51: 716-723, 2015.

21. Berwouts D, Swimberghe M, Duprez F, Boterberg T, Bonte K, Deron P, De Gersem W, De Neve W and Madani I: Intensity-modulated radiotherapy for early-stage glottic cancer. Head Neck 2014 (Epub ahead of print).

22. Janssen S, Glanzmann C, Huber G and Studer G: Risk-adapted partial larynx and/or carotid artery sparing modulated radiation therapy of glottic cancer. Radiat Oncol 9: 136, 2014.

23. Boomsma MJ, Bijl HP, Christianen ME, Beetz I, Chouvalova O, Steenbakkers RJ, van der Laan BF, Wolffenbuttel BH, Oosting SF, Schilstra C and Langendijk JA: A prospective cohort study on radiation-induced hypothyroidism: Development of an NTCP model. Int J Radiat Oncol Biol Phys 84: e351-e356, 2012.

24. Nishi T, Nishimura Y, Shibata T, Tamura M, Nishigaito N and Okumura M: Volume and dosimetric changes and initial clinical experience of a two-step adaptive intensity modulated radiation therapy (IMRT) scheme for head and neck cancer. Radiother Oncol 106: 85-89, 2013. 